\title{
Solution of the Second Order Linear Differential Equation
}

\author{
E. J. Kanellopoulos, Th. V. Kanellopoulos, and K. Wildermuth \\ Institut für Theoretische Physik der Universität Tübingen
}

Received February 12/October 15, 1971

\begin{abstract}
An integro-iteration method is applied to solve the second order linear differential equation. Although the procedure is quite elementary it produces the general solution in form of a simple series which can be made to converge absolutely, at least in the region of interest. Particular solutions with given initial or boundary values can be readily obtained from the general solution.
\end{abstract}

\section{Introduction}

In this note we present an elementary method for deriving the general solution, or any particular one with given boundary conditions, of a second order linear differential equation, in a relatively simple form and, most important, convenient both for analytical studies and for numerical applications.

Basically the method used is an iterative one into - which a step of integration has been inserted - thus the name "integro-iteration" procedure. By this way one gets the general solution. From it the derivation of any solution with given boundary conditions is straightforward.

We believe that for the solution of a second order linear differential equation, the present method, at least for practical applications, is preferable than the existing general ones, for the following reasons:

(i) The method is elementary and thus accessible to anybody with only basic knowledge of mathematics.

(ii) It is quite flexible, as one has complete freedom in choosing the unperturbed equation and also a large choice for its partial solutions. This is quite helpful in fitting the solution to the conditions of the problem, and

(iii) The solutions are obtained in form of a simple series which can be made to converge absolutely, faster than an exponential one, at least in the region of interest. To make clear this point, consider the case of a boundary value problem. One applies e.g. Fredholm's method, and has to compute two series. Each of these series is a multiple one, i.e. the $n^{\text {th }}$ 
term has $n$ ! components, while in the present method each term of the series has only one component.

Applications of the method will appear in a series of forthcoming papers, the first dealing with the solution of the Schrödinger equation for central potential, under rather weak restrictions on it, both for scattering and for bound states problems.

\section{The Method. Solution of Type I}

Suppose we want to find the general solution of the equations

$$
z^{\prime \prime}+A(z) z=0
$$

around a point $x=a$.

We write (I) as

$$
z^{\prime \prime}+B(x) z=C(x) z
$$

where $B(x)$ is such that the "unperturbed" equation

$$
u^{\prime \prime}+B(x) u=0
$$

is readily soluble.

Let $u_{1}$ and $u_{2}$ be two independent solutions of (3), normalised in such a way that:

$$
W\left(u_{1}, u_{2}\right)=u_{1} u_{2}^{\prime}-u_{1}^{\prime} u_{2}=1 .
$$

Using $u_{1}$ and $u_{2}$ we can put Eq. (2) under the form:

$$
z=u_{1}\left[\kappa_{1}-\int^{x} C u_{2} z d x^{\prime}\right]+u_{2}\left[\kappa_{2}+\int^{x} C u_{1} z d x^{\prime}\right]
$$

with $\kappa_{1}$ and $\kappa_{2}$ arbitrary constants.

Now we split $z$ as follows:

$$
\begin{aligned}
& w_{1}=u_{1}\left[\kappa_{1}-\int^{x} C u_{2}\left(w_{1}+w_{2}\right) d x^{\prime}\right], \\
& w_{2}=u_{2}\left[\kappa_{2}+\int^{x} C u_{1}\left(w_{1}+w_{2}\right) d x^{\prime}\right], \\
& w_{1}+w_{2}=z .
\end{aligned}
$$

For the iteration we write

$$
\begin{aligned}
& w_{1}^{(n)}=u_{1}\left[\kappa_{1}-\int^{x} C u_{2}\left(w_{1}^{(n)}+w_{2}^{(n)}\right) d x^{\prime}\right], \\
& w_{2}^{(n)}=u_{2}\left[\kappa_{2}+\int^{x} C u_{1}\left(w_{1}^{(n-1)}+w_{2}^{(n)}\right) d x^{\prime}\right] .
\end{aligned}
$$


At this point we insert the integration step, i.e. instead of applying the iteration using Eqs. (7a) and (7b) as they stand, we integrate them first, as follows. In Eq. (7a) we consider $w_{2}^{(n)}$ to be known and integrate with respect to $w_{1}^{(n)}$, and in Eq. (7b) we consider $w_{1}^{(n-1)}$ as known and integrate with respect to $w_{2}^{(n)}$. In this way we obtain

$$
\begin{aligned}
w_{1}^{(n)} & =u_{1} e^{-f(x)}\left[c_{1}-\int_{a}^{x} C u_{2} e^{f\left(x^{\prime}\right)} w_{2}^{(n)} d x^{\prime}\right], \\
w_{2}^{(n)} & =u_{2} e^{f(x)}\left[c_{2}+\int_{a}^{x} C u_{1} e^{-f\left(x^{\prime}\right)} w_{1}^{(n-1)} d x^{\prime}\right], \\
f(x) & =\int^{x} C u_{1} u_{2} d x^{\prime} .
\end{aligned}
$$

Note that the lower limit of integration in $f(x)$ is irrelevant.

Starting with $w_{1}^{(0)}=u_{1} e^{-f(x)}$ we, finally, find:

$$
\begin{aligned}
w_{1}(x)=u_{1} e^{-f(x)}\left[c_{1} \Phi_{1}\left(\begin{array}{c}
x \\
a, a
\end{array}\right)-c_{2} \int_{a}^{x} C u_{2}^{2} e^{2 f} \Phi_{2}\left(\begin{array}{c}
x_{1} \\
a, a
\end{array}\right) d x_{1}\right], \\
w_{2}(x)=u_{2} e^{f(x)}\left[c_{1} \int_{a}^{x} C u_{1}^{2} e^{-2 f(x)} \Phi_{1}\left(\begin{array}{c}
x_{1} \\
a, a
\end{array}\right) d x_{1}+c_{2} \Phi_{2}\left(\begin{array}{c}
x \\
a, a
\end{array}\right)\right] \\
\Phi_{1}\left(\begin{array}{c}
x \\
a, a
\end{array}\right)=1+\sum_{n=1}^{\infty}(-)^{n}\left\{q_{1}(x)\right\}_{n}, \\
\Phi_{2}\left(\begin{array}{c}
x \\
a, a
\end{array}\right)=1+\sum_{n=1}^{\infty}(-)^{n}\left\{q_{2}(x)\right\}_{n}, \\
q_{1}(x)=\int_{a}^{x} C u_{2}^{2} e^{2 f} d x_{1} \int_{a}^{x_{1}} C u_{1}^{2} e^{-2 f} d x_{2}, \\
q_{2}(x)=\int_{a}^{x} C u_{1}^{2} e^{-2 f} d x_{1} \int_{a}^{x_{1}} C u_{2}^{2} e^{2 f} d x_{2} .
\end{aligned}
$$

By $\{q\}_{n}$ we mean the $2 n$-dimensional integral formed by $n$ repetitions of the double integral $q$. For example

$$
\left\{q_{1}\right\}_{2}=\int_{a}^{x} C u_{2}^{2} e^{2 f} d x_{1} \int_{a}^{x_{1}} C u_{1}^{2} e^{-2 f} d x_{2} \int_{a}^{x_{2}} C u_{2}^{2} e^{2 f} d x_{3} \int_{a}^{x_{3}} C u_{1}^{2} e^{-2 f} d x_{4} \text { e.t.c. }
$$

The question of convergence of the series (10) is discussed in Part III. The general solution $z=w_{1}+w_{2}$ is:

$$
\begin{aligned}
z= & c_{1}\left[u_{1} e^{-f} \Phi_{1}\left(\begin{array}{c}
x \\
a, a
\end{array}\right)+u_{2} e^{f} \int_{a}^{x} C u_{1}^{2} e^{-2 f} \Phi_{1}\left(\begin{array}{c}
x_{1} \\
a, a
\end{array}\right) d x_{1}\right] \\
& +c_{2}\left[-u_{1} e^{-f} \int_{a}^{x} C u_{2}^{2} e^{2 f} \Phi_{2}\left(\begin{array}{c}
x_{1} \\
a, a
\end{array}\right) d x_{1}+u_{2} e^{f} \Phi_{2}\left(\begin{array}{c}
x \\
a, a
\end{array}\right)\right] .
\end{aligned}
$$


Two partial solutions of Eq. (2) are:

$$
\begin{aligned}
& z_{1}=u_{1} e^{-f(x)} \Phi_{1}\left(\begin{array}{c}
x \\
a, a
\end{array}\right)+u_{2} e^{f(x)} \int_{a}^{x} C u_{1}^{2} e^{-2 f} \Phi_{1}\left(\begin{array}{c}
x_{1} \\
a, a
\end{array}\right) d x_{1}, \\
& z_{2}=u_{2} e^{f(x)} \Phi_{2}\left(\begin{array}{c}
x \\
a, a
\end{array}\right)-u_{1} e^{-f(x)} \int_{a}^{x} C u_{2}^{2} e^{2 f} \Phi_{2}\left(\begin{array}{c}
x_{1} \\
a, a
\end{array}\right) d x_{1} .
\end{aligned}
$$

It is a matter of simple algebra to show that Eqs. (13) satisfy Eq. (2) as,

$$
\begin{aligned}
& \Phi_{1}\left(\begin{array}{c}
x \\
a, a
\end{array}\right)=1-\int_{a}^{x} C u_{2}^{2} e^{2 f} d x_{1} \int_{a}^{x_{1}} C u_{1}^{2} e^{-2 f} \Phi_{1}\left(\begin{array}{c}
x_{2} \\
a, a
\end{array}\right) d x_{2} \\
& \Phi_{2}\left(\begin{array}{c}
x \\
a, a
\end{array}\right)=1-\int_{a}^{x} C u_{1}^{2} e^{-2 f} d x_{1} \int_{a}^{x_{1}} C u_{2}^{2} e^{2 f} \Phi_{2}\left(\begin{array}{c}
x_{2} \\
a, a
\end{array}\right) d x_{2} .
\end{aligned}
$$

The solutions (13a) and (13b) are independent as their Wronskian is equal to 1 .

We note that to each of the partial solutions corresponds one of the functions $\Phi_{1}$ and $\Phi_{2}$. These functions $\Phi_{1}$ and $\Phi_{2}$ are called "central" functions for the solution of the Eq. (2).

\section{Solution of Type II}

Consider the pair of functions:

$$
\begin{aligned}
& H_{1}=e^{-f(x)} \Phi_{1}\left(\begin{array}{c}
x \\
a, a
\end{array}\right)=e^{-f}\left[1-\int_{a}^{x} C u_{2}^{2} e^{2 f} d x_{1} \int_{a}^{x_{1}} C u_{1}^{2} e^{-2 f} \Phi_{1} d x_{2}\right] \\
& \text { and } \quad F_{1}\left(\begin{array}{c}
x \\
a, a
\end{array}\right)=1+\int_{a}^{x} C u_{2}^{2} d x_{1} \int_{a}^{x_{1}} \frac{F_{1}\left(\begin{array}{c}
x_{1} \\
a, a
\end{array}\right)}{u_{2}^{2}} d x_{2} .
\end{aligned}
$$

One finds that the functions $u_{2} \sqrt{C} \cdot H_{1}$ and $u_{2} \sqrt{C} \cdot F_{1}\left(\begin{array}{c}x \\ a, a\end{array}\right)$ fulfill the same differential equation, i.e.

$$
y^{\prime \prime}+\left\{\frac{\left(C u_{2}^{2}\right)^{\prime \prime}}{2 C u_{2}^{2}}-\frac{3}{4}\left[\frac{\left(C u_{2}^{2}\right)^{\prime}}{C u_{2}^{2}}\right]^{2}-C\right\} y=0
$$

and therefore their Wronskian is constant

$$
W\left(u_{2} \sqrt{C} \cdot H_{1}, u_{2} \sqrt{C} \cdot F_{1}\right)=C u_{2}^{2} W\left(H_{1}, F_{1}\right)=\text { const. }
$$

If we choose in Eqs. (15) the limit $a$ such that $u_{1}(a)=0$ and put $f=\int_{a}^{x} C u_{1} u_{2} d x^{\prime}$, this Wronskian is zero and $H_{1} \equiv F_{1}$, or

$$
\Phi_{1}\left(\begin{array}{c}
x \\
a, a
\end{array}\right)=e^{\int^{x} C u_{1} u_{2} d x^{\prime}} F_{1}\left(\begin{array}{c}
x \\
a, a
\end{array}\right) .
$$


Inserting Eq. (16) into Eq. (13a), after some integrations by parts we derive:

$$
z_{1}=-u_{2} \int_{a}^{x} \frac{F_{1}\left(\begin{array}{c}
x^{\prime} \\
a, a
\end{array}\right)}{u_{2}^{2}} d x^{\prime}
$$

where $F_{1}\left(\begin{array}{c}x \\ a, a\end{array}\right)$ is given by (15b).

This is another expression for the solution $z_{1}$, when it fulfills the condition

$$
z_{1}(a)=0
$$

Analogous expressions we can derive for the solution $z_{2}$.

\section{Convergence}

If we have generally

$$
q=\int_{a}^{x} \varphi_{1} d x_{1} \int_{a}^{x_{1}} \varphi_{2} d x_{2}
$$

it is easy to prove that

$$
\begin{aligned}
& \left|\{q\}_{n}\right| \leqq \frac{\bar{q}^{n}}{n !} \\
& \bar{q}=\int_{a}^{x}\left|\varphi_{1}\right| d x_{1} \int_{a}^{x_{1}}\left|\varphi_{2}\right| d x_{2} .
\end{aligned}
$$

where

For example

$$
\begin{aligned}
\left|\{q\}_{2}\right|= & \left|\int_{a}^{x} \varphi_{1} d x_{1} \int_{a}^{x_{1}} \varphi_{2} d x_{2} \int_{a}^{x_{2}} \varphi_{1} d x_{3} \int_{a}^{x_{3}} \varphi_{2} d x_{4}\right| \\
& \leqq \int_{a}^{x}\left|\varphi_{1}\right| d x_{1} \int_{a}^{x_{1}}\left|\varphi_{2}\right| d x_{2} \int_{a}^{x_{2}}\left|\varphi_{1}\right| d x_{3} \int_{a}^{x_{3}}\left|\varphi_{2}\right| d x_{4} \\
& <\int_{a}^{x}\left|\varphi_{1}\right| d x_{1} \int_{a}^{x_{1}}\left|\varphi_{2}\right| d x_{2} \int_{a}^{x_{1}}\left|\varphi_{1}\right| d x_{3} \int_{a}^{x_{3}}\left|\varphi_{2}\right| d x_{4} \\
= & \frac{\left[\int_{a}^{x}\left|\varphi_{1}\right| d x_{1} \int_{a}^{x_{1}}\left|\varphi_{2}\right| d x_{2}\right]^{2}}{2 !}=\frac{\bar{q}^{2}}{2 !}
\end{aligned}
$$

$$
x \geqq x_{1} \geqq x_{2} \geqq x_{3} \geqq a \quad\left(\text { or } x \leqq x_{1} \leqq x_{2} \leqq x_{3} \leqq a\right) .
$$


Therefore a sufficient condition for the convergence of a series of the type $\sum_{n=0}^{\infty}( \pm)^{n}\{q\}_{n}$ is that $\bar{q}$ given by (19), is finite.

Returning to our case we have that sufficient conditions for the convergence of $\Phi_{1}\left(\begin{array}{c}x \\ a, a\end{array}\right)$ and $F_{1}\left(\begin{array}{c}x \\ a, a\end{array}\right)$ are respectively:

$$
\begin{aligned}
& \int_{a}^{x}\left|C u_{2}^{2} e^{2 f}\right| d x_{1} \int_{a}^{x_{1}}\left|C u_{1}^{2} e^{-2 f}\right| d x_{2}<M<\infty, \\
& \int_{a}^{x}\left|C u_{2}^{2}\right| d x_{1} \int_{a}^{x_{1}}\left|\frac{1}{u_{2}^{2}}\right| d x_{2}<N<\infty .
\end{aligned}
$$

Similar conditions are for $\Phi_{2}\left(\begin{array}{c}x \\ a, a\end{array}\right)$ and $F_{2}\left(\begin{array}{c}x \\ a, a\end{array}\right)$.

If $u_{1}$ and $u_{2}$ are real, $u_{1}(a)=0$, and if in the interval $(a, x)$ no zeros of $u_{2}$ exist, then we have

$$
\int_{a}^{x}\left|C u_{2}^{2}\right| d x_{1} \int_{a}^{x_{1}}\left|\frac{1}{u_{2}^{2}}\right| d x_{2}=\int_{a}^{x}\left|C u_{1} u_{2}\right| d x_{1} .
$$

In this case the condition for the convergence of $F_{1}\left(\begin{array}{c}x \\ a, a\end{array}\right)$ and $\Phi_{1}\left(\begin{array}{c}x \\ a, a\end{array}\right)$ is:

$$
\int_{a}^{x}\left|C u_{1} u_{2}\right| d x_{1}<\mu<\infty .
$$

\section{Application to Problems with Boundary Conditions}

\section{The Homogeneous Equation}

a) Initial value problems. The conditions on the solution $z$ can be reduced to, $z(a)=0$ and $z^{\prime}(a)=\lambda^{1}$.

To obtain this solution it is enough to choose one of $u_{1}$ and $u_{2}$ to fulfill the same conditions i.e.

$$
\begin{aligned}
& u_{1}(a)=0, \\
& u_{1}^{\prime}(a)=\frac{1}{u_{2}(a)}=\lambda .
\end{aligned}
$$

Then either (13a) or (17) represent the required solution.

b) Boundary value problems. Here we can reduce the requirements of the problem to the conditions

$$
\begin{aligned}
& z(a)=0, \\
& z(b)=0 .
\end{aligned}
$$

1 This condition is not necessary, as only the first one defines the solution apart from a factor which is irrelevant for homogeneous equations. 
Choosing $u_{1}$ and $u_{2}$ such that

$$
\begin{aligned}
& u_{1}(a)=0 \\
& u_{2}(b)=0
\end{aligned}
$$

we see that condition (23a) is satisfied for (13a) or (17). In order that also condition (23b) is fulfilled we must have

$$
\begin{array}{ll}
\text { for Eq. (13a) } & \Phi_{1}\left(\begin{array}{c}
b \\
a, a
\end{array}\right)=0, \\
\text { for Eq. (17) } & F_{1}\left(\begin{array}{c}
b \\
a, a
\end{array}\right)=0 .
\end{array}
$$

These are the eigenvalue equations. We note that because of (16) these are equivalent.

If instead of (13a) we used (13b) we find as eigenvalue equation

$$
\Phi_{2}\left(\begin{array}{c}
a \\
b, b
\end{array}\right)=0
$$

but it is obvious that

$$
\Phi_{1}\left(\begin{array}{c}
b \\
a, a
\end{array}\right)=\Phi_{2}\left(\begin{array}{c}
a \\
b, b
\end{array}\right)
$$

Any parameter existing in Eq. (1) can be used to derive eigenvalues from Eq. (24)

\section{The Inhomogeneous Equation}

$$
z^{\prime \prime}+A(x) z=D(x) .
$$

As it is known the general solution is:

$$
z=c_{1} z_{1}+c_{2} z_{2}-z_{1} \int_{a}^{x} D z_{2} d x_{1}+z_{2} \int_{a}^{x} D z_{1} d x_{1}
$$

where $z_{1}$ and $z_{2}$ are two independent solutions of the homogeneous equation and $c_{1}$ and $c_{2}$ arbitrary constants.

The procedure to find special solutions with fixed boundary conditions is quite trivial. We mention here only the case of a boundary value problem in which we demand

$$
z(a)=0, \quad z(b)=0
$$

and it happens $\Phi_{1}\left(\begin{array}{c}b \\ a, a\end{array}\right)=0\left(\right.$ or $\left.F_{1}\left(\begin{array}{c}b \\ a, a\end{array}\right)=0\right)$. 
By choosing $u_{1}$ and $u_{2}$ such that

$$
u_{1}(a)=0, \quad u_{2}(b)=0
$$

we find that the conditions for the existence of the solution is

$$
\int_{a}^{b} D z_{1} d x=0
$$

which expresses the third Fredholm's theorem. Then the solution is not uniquely defined. We obtain

$$
z=z_{1}\left[c-\int_{a}^{x} D z_{2} d x_{1}\right]+z_{2} \int_{a}^{x} D z_{1} d x_{1}
$$

where $c$ is arbitrary constant.

Acknowledgement. One of us (T.V.K.) would like to thank the Deutsche Forschungsgemeinschaft for a grant.

Th. V. Kanellopoulos Institut für Theoretische Physik der Universität Tübingen

D-7400 Tübingen, Köstlinstr. 6 